\title{
Erratum to: Effects of vertical muscle surgery on differences in the orientation of Listing's plane in patients with superior oblique palsy
}

\author{
Manabu Miyata • Yoshie Shira • Reika Kono • \\ Takashi Furuse • Ichiro Hamasaki • Satoshi Hasebe • \\ Hiroshi Ohtsuki
}

Published online: 3 December 2013

(C) Springer-Verlag Berlin Heidelberg 2013

Erratum to: Graefes Arch Clin Exp Ophthalmol

(2013) 251:2437-2443

DOI 10.1007/s00417-013-2407-3

The original version of this article inadvertently contained mistake.

Figure legends 3

wrong: The triangles indicate the mean preoperative data and the circles indicate the mean postoperative data $(n=7)$.

right: The triangles indicate the mean postoperative data and the circles indicate the mean preoperative data $(n=7)$.

Figure legends 4

wrong: The triangles indicate the mean preoperative data and the circles indicate the mean postoperative data $(n=3)$.

right: The triangles indicate the mean postoperative data and the circles indicate the mean preoperative data $(n=3)$.

The online version of the original article can be found at http://dx.doi.org/ 10.1007/s00417-013-2407-3.

M. Miyata $(\bowtie) \cdot$ Y. Shira $\cdot$ R. Kono $\cdot$ T. Furuse $\cdot$ I. Hamasaki $\cdot$

S. Hasebe $\cdot$ H. Ohtsuki

Department of Ophthalmology, Okayama University, Graduate

School of Medicine, Dentistry, and Pharmaceutical Sciences, 2-5-2

Shikata-cho, Kita-ku, Okayama 700-8558, Japan

e-mail: miyat-m@cc.okayama-u.ac.jp 